\title{
The Universe Is Otherwise/External Gravity
}

\section{Paul Schroeder}

Wind Lake, WI, USA

Correspondence to: Paul Schroeder,Pshrodr8@aol.com

Keywords: Gravity, EM Radiation/Light, Universe, Rotation, Revolution, Doppler, Infinity, Electrons, Matter, Magnetism, Bumps, Barycenter, Black Holes

Received: July 12, $2021 \quad$ Accepted: September 6, $2021 \quad$ Published: September 9, 2021

Copyright $\odot 2021$ by author(s) and Scientific Research Publishing Inc.

This work is licensed under the Creative Commons Attribution International License (CC BY 4.0).

http://creativecommons.org/licenses/by/4.0/

\section{Open Access}

\section{ABSTRACT}

The ideas that follow provide a totally different view of the universe. These are perspectives by which to view everything without clashing over technical understandings. Sufficient examination of this model should open minds and add enhanced views for those uncomfortable with current cosmology.

\section{THE SIGNIFICANT DIFFERENCES OF THIS COSMOLOGY ARE}

1) Current expansion theory originally came from, and absolutely needs, stellar redshifts to be caused by Doppler motion away by all bodies. That explanation for redshift changed all astrodynamics and led to the standard model of physics. But the redshift can also occur when there is relative sideways (perpendicular) motion by the source relative to the observer. One explanation of this is in a Wikipedia article on Doppler-Doppler and the End of Expansion [1,2].

All understanding of the universe depends on which Doppler is chosen to define space as being steady or expanding. It has been the impractical choice of redshift caused by motion away that leads our thoughts. Science chose the unmeasurable or visible speculative motion away-over the obvious visible relative rotation of stars to us-as the cause of redshift.

2) The universe is infinite but physics chose otherwise. Their finite universe and having borders gained belief when supported by the church. The big bang idea is for playing, not for understanding.

3) Extra dimensions are further imagination play. Too many models are metaphysics.

4) Space has a real nature, and that nature is EM radiation. All debate of radiation's nature between wave and matter succumbs to accepting "radiation flows". Anything that moves causes pressure. Empty space could never move nor exist.

5) Newton's empty space and singular direction (downward) of gravity beams is illogical. It requires other infinite sideways motion, thus ignoring the need for some drive source. Newton's theory deduces that gravity is significantly caused only by matter.

6) We say gravity is real and pushes rather than attracts. Note, attracting is a pull and pull is not a physics function. The push is from EM radiation and that is the source of gravity. Gravity push comes from all X Y Z directions, even serving to hold all things together. The "attraction" we experience is the 
offset of incoming gravity beams being offset by diminished beams exiting up from the matter. Resulting gravity is experiencing the "net" force.

7) EM radiation penetrates matter. Matter affects that radiation by diminishing its push energy into heat and light. The matter applies its rotation of the sphere to the exiting beams of radiation causing a swirl in the local atmosphere. Also, the maximum heat and maximum downward push are generated at the surface where the net pressures differ most between incoming and exiting waves. Space around a globe is swirling due to the globe's rotation. That swirl causes a lateral pressure on orbitals and pushes them in their orbits.

8) All stars and planets rotate. The atmospheres of two nearby rotating stars push each other and they rotate around the central point called a barycenter [3].

By extension a center can apply for numerous or millions of stars including the central point of a galaxy center. The more the stars participating the more the spin. Being all swirl rather than being matter, the center gives the impression of a black hole when viewed from the plane of the swirls. Black holes are simply intense rotation centered in galaxies caused by its many rotating stars. The rotation seen from along the galaxy plane is black, and views from the $\mathrm{z}$ axis above would suggest a doughnut. We see the rotation of the nature of space. Matter is not a component of black holes.

9) Light radiated from a body is affected/slowed by gravity of the source body throughout its travels. This effect is so trivial it is usually ignored, but it does continue throughout the flow of light. Thus the light beam gains redshift due to gravity, however trivially. A receiving body subsequently attracts the light, partially offsetting the red shift with some blue shift due to faster flow of light.

The Pound-Rebka experiment, using a tower, demonstrated that the velocity difference (acceleration) of photons is "identical to that which a material object would acquire in free fall", as predicted by Newton's emission theory of light. [4]

Support to the Pound Rebka experiment includes light sent to earth from a rocket that proved blue shift by the pull of destination bodies. Given any accumulating redshifts, light from extreme distances is redshifted into microwaves and beyond. Without the red shifting of light our night would be fully lighted. Stars exist at infinite distances.

10) Overall EM radiation is in balance throughout the universe. Beams redshifted by gravity can be offset a rebuilding of the frequencies toward the blue when EM radiation penetrates matter.

\section{SOME OBSERVATIONS}

1) Magnetism is the result of some gravity beams being redirected, usually by 90 degrees. An electromagnet gives an example as activated electrons push incoming gravity beams sideways, causing an imbalance of push pressure. More push pressure resides at one end point of the magnet while less gravity beams and thus less pressure resides at the opposite point.

2) Since gravity beams can be redirected then within a sphere of space action missing from some direction(s) can experience anti-gravity, i.e. blocked gravity. Pushing and/or lifting energy is assisted.

3) Electrons of atomic matter are simply the crossing of opposite direction high frequency EM beams. Any direction of 90 degree or more causes a crossing of waves that begins the creation of matter. The up and down flow of the opposing wavelengths define the electron charge. Protons and neutrons are regions in the matter. The charge depends on the region's location relative to the electron crossing.

4) Matter is continuously created as incoming EM beams intersect existing waves or initial matter in an atmosphere. The closer to the sphere the more crossings occur, modifying matter.

5) The EM spectrum becomes a "Spectrum of Existence" as we learn that long wave beams function as gravity. Shorter length high frequency waves interact via crossings leading to matter being created and so the matter depends on very short waves.

6) Kepler's third law relates the motions of orbitals such as planets around the sun to each other. The law is "the square of the orbital period of a planet is directly proportional to the cube of the semi-major 
axis of its orbit”. $\mathrm{P}^{\wedge} 2=\mathrm{D}^{\wedge} 3 \times \mathrm{k}$. There is no known reason for this relationship though it seems it must be caused by the suns rotation. Nothing about the sun's actions or its equator provides an obvious cause for the pattern of orbital times and distances. But realize that the atmospheric rotation near the sun is one direction due to its rotation while more distant revolutions of planets seem to be in the opposite direction. The direction changes for what would be the "synchronous orbit, like earth's geosynchronous orbit". "Where orbit matches rotation of center body", rotation causes the pattern for the sun or whatever central body is used (such as Jupiter). The relative planet distances then begin to match each other beyond that orbit and the synchronous distance of that orbit for the sun should be at about 11 diameters.

\section{DETAILS FUNDAMENTAL DETAILS OF EXTERNAL GRAVITATION}

1) The term gravity can refer to the functional source or to the detectible effect of the action of the source. The effects are motion and the user of motion which implies existence of matter.

Gravity is a push rather than an attraction. As such it solves "action at a distance".

The push implies the source is external from matter. The source is beams with velocity. Motion of beams is confirmed by waves within the beams.

2) 'My 'PAEP' was something I had devised a couple decades ago in answer to the distinction of particles vs waves for scientific explanations. Throughout centuries from the pushing theories of Le Sage and Fatio to Ideas of Einstein, physics speculated with a particle labeled 'graviton' for explaining gravity as well as other purposes. To separate and identify its purpose, I replaced graviton with my PAEP which is a 'Particle that Applies External Pressure'. The distinction of wave vs. particle has continuously confused science as only particle actions use mathematical pressure measures. In fact, measures have shown pressures from various wave length beams, including light, upon impacting matter. Due to science focus upon attraction, a feature must cover pushing pressure. I then had to go beyond particle and use the wave within beams/rays of radiation to push as my pushing gravity. That diminished my use of PAEP and led to a focus on the interactions of waves and their frequency and altitude which relate to the push. Someday the PAEP may return as a much smaller particle defining waves."

3) Gravitation pushes as if it contained moving particles-Paeps-"particles applying external pressure". But gravity particles can't be matter. They would cause too much heat upon impact and would interfere with orbiting.

Gravitation is best pictured as lines rather than fields. Beam lines help analyze and contemplate a linear push. Pressure gradients that summarize the situation inhibit analysis.

Gravitation functions as beams pushing from all directions upon every point of space. Thus matter takes on spherical shapes. Attraction gravity is linear and insufficient to understand the universe. We need transverse radiation wave/particles that strike matter with impact. The amount of impact depends on the wave frequency and altitude. Light and EM radiation are composed of their waves which are their geometric pressure causing particles. We dispose of the concept of photons and of science's wave/matter conflict.

4) EM radiation rarely or slightly penetrates masses due to its wave structure. But longer wave gravity radiation penetrates more and extends its push throughout. Thus Paep gravity beams replicate long wave EM radiation.

Gravity beams, like radiation waves, move at velocity "C". Gravity carries radiation.

5) The universe is infinite and isotropic. Space contains EM waves but does not contain matter particles. Gravity beams are the structure of space simulating the aether others refer to. Like-wise gravity is the undetectable background.

6) Gravity interacts with matter. Matter exists as rotations/spins relative to the local equilibrium. The spin may be of the atomic particles such as protons and electrons as well as spin of the entire mass. The amount of spin determines the density of masses. The lack of spin signifies the absence of matter.

7) Equilibrium is the balance of vertical pushes, yet with an imbalance of horizontal pushes.

8) Gravity beams mostly penetrate matter. Atoms are mostly empty space. Paep waves interacting with atomic particles modify each other, leading to heat and light. 
Penetrating gravity beams exit the mass and are modified. Beams may be diminished, their wavelength shortened and modified into heat and light radiation, and/or have its motion redirected.

The gravitational push at a mass surface offsets diminished exiting beams with undiminished incoming beams resulting in a "NET" downward push often called attraction gravity.

9) $\mathrm{R} \wedge 2$ laws work for attraction gravity because it is centered at a point. Pushing gravity pushes all $3 \mathrm{X}$ $\mathrm{Y} \mathrm{Z}$ directions leading to the same central point of reference for equations.

10) During gravity penetration impacted matter particles are modified into radiation or different matter. The sun's eternal power is caused by continuous penetration and exiting of gravity beams whose frequency has been increased prior to exiting.

11) Paeps can be redirected by spin of atomic particles or by the spin of the whole mass. Spin defines matter because the moving mass particles intersect incoming gravity beams.

Redirected Paep beams exit in a bent path relative to both the source and the observer. They curve throughout their travels.

12) Space is 3 dimensional but its contents flow and distort linear analyses.

13) Undiminished gravity beams flow in all directions across a mass surface, not just downward. A counterclockwise flow of exiting beams bent by the rotation of the mass occurs. The flow velocity matches the mass's rotation yielding local equilibrium.

14) Counterclockwise motion relative to our $Z$ axis north and to orbital centers dominates the spin and orbital motions of the universe. Antigravity is simply a clockwise flow.

15) Newton said orbital motion continues absent external forces, thus no friction. The implied void of space can't exist given radiation, meteors, and solar winds. A motive force is needed.

Newton's "motive" external force was centrifugal force, subsequently inertia, a force with no source. Newton's inertia is more properly defined as "adhering to the local flow of gravity". The application of inertia saved Newton from explaining the source of motions.

16) Planets incur lateral pushes of gravity caused by the bent beams from the sun' Likewise moons incur pushes from the sun and their planet. This is the motive force causing orbiting. The revolution push by spinning bodies upon their orbitals decreases with altitude increase.

17) Orbital revolution rates must be less than their central body rotation rate.

18) The lateral pushes on orbitals cause the rotation of the orbital as well as the revolution. The rotation rate is dependent on how far to the right of the 12 o'clock/6 o'clock axis the push is centered.

19) Rotating bodies usually rotate counterclockwise relative to their central body. Central body bent gravity beams add to atmospheric as well as the masses "rotation" for orbitals. The sum of bent gravity beams from earth's rotation and from the sun causes winds on earth.

20) Solar gravity beams are the solar wind when passing by earth. Magnetosphere pictures are attempts to represent bent gravity beams. At a point between planet and the sun, bent beams from each will collide, creating a small void.

21) The nuclear-strong force is simply the sum of gravitation pushing from all directions.

22) Magnetism is the "net" push of gravity beams when beams from one direction are redirected.

23) Charge is simply the direction of flow. Anti-gravity is pushing in the opposite direction.

24) A spectrum of existence associates waves from the longest Paep gravity beams, thru EM radiation, and extending to "short wave" mass itself. All relate to the wave structure in the spectrum of EM radiation.

25) Three dimensional waves are best pictured as coils. Consider a flowing beam wrapping around inside a straw like a counterclockwise screw.

26) Diminished gravitation occurring locally within the sun or stars is replenished by the gravitational stretching of light beams into microwaves, then radio waves, and further into Paep gravity beams as they travel from very distant stars.

27) Rotating bodies cause orbitals to encircle them. Kepler detected this and determined orbital times for our solar system with a large central body. More nearly equal bodies are not similarly studied and having joint revolution sources significantly changes/decreases the orbital times calculations. Gravity is not 
different in galaxies.

28) Kepler's third law is that the inner planets revolution period is a constant, times the $3 / 2$ power of the solar distance $\mathrm{Rn}$ for each planet. Thus, the central body sun has to be the source of their orbital times. It is the push by bent solar gravity beams that forms this relationship as the orbits increase by $\mathrm{R}^{\wedge} 2$. The factor $\mathrm{R}$ is the 2 -dimensional component, and $\mathrm{R}^{\wedge} 1 / 2$ is the $\mathrm{Z}$ axis contribution.

29) The bent flow of gravity defeats exact linear calculations in large geometric analyses. The difficulty increases when multiple rotating masses contribute to the flow.

30) Actions within our solar system contend simultaneously with the linear flow of space at speed C $(300,000 \mathrm{Km} / \mathrm{sec})$ and the perpendicular flow from the solar rotation of $20 \mathrm{Km} / \mathrm{sec}$. This gives the bending of solar gravity beams.

31) Sufficient bending of radiation beams and interaction with other beams creates mass. Electrons are beam crossings.

32) Gravity beams condense together approaching mass bodies and the beams interact such that crossings are electrons. The regions created are protons and neutrons. Thus the higher frequency EM waves can create matter. Two EM beams may create matter if their waves intersect. The wave crossing is the electron, having spin, due to the 2 beams flow directions, and has no real weight. The spin is plus or minus based on the wave directions which defines proton vs neutron.

Electrons remain in place relative to the nucleus, not rotating.

Multiple crossings of waves create more massive masses.

Beams entering the atmosphere can intersect with others and the closer to ground the more complex the element created or converted.

33) Regions of matter creation occur when 2 beams from 120 degree angles interact. The Trojan asteroids are where solar and Jupiter EM radiation beams interact.

34) A proper picture of gravity beams diminished by the sun and traveling toward earth are bent inward from the right. The motion of earth causes unbent beams to arrive from the left as if bent by earth's motion.

35) Black holes are product of multiple stars whose spins interact at a common point-barycenter. Black holes are interacted spins on a common plane.

36) All masses including the earth grew gradually as EM interactions created electron crossings and formed new and/or more complex elements.

37) The complex high numbered elements on earth were created here! and are results of EM interactions within mass bodies (earth).

We reject what standard model says, elements come from gas flows from star explosions, or from black holes.

38) EM radiation waves may lose frequency due to gravity and they gain frequency when penetrating matter. Such gives equilibrium of the universe.

39) Much attention has been given to an odd solar event called "Gravity Bumps during Eclipses" [5] The Earth's "bending" of gravity beams produces distortion to both sides of the light blackout area on the moon is called the bumps of gravity and is detected during a lunar eclipse.

My cosmology says "The gravity beams from the sun pass earth on all sides in slightly bent flows. Upon arriving at the eclipsing moon the gravity beams bend causes distortion to arrive before the light changes while at the end of the eclipse the gravity changes after the light change".

\section{CONFLICTS OF INTEREST}

The author declares no conflicts of interest regarding the publication of this paper.

\section{REFERENCES}

1. Wikipedia (n.d.) Relativistic Doppler Effect From. https://en.wikipedia.org/wiki/Relativistic Doppler effect

2. Schroeder, P. (2021) Doppler and an End of Expansion. Crimson Publishers, Los Angeles. 
3. NASA Space Place-NASA Science for Kids. What Is a Barycenter? https://spaceplace.nasa.gov/barycenter/

4. Explanation of the Pound Rebka Experiment. http://www.anti-relativity.com/poundrebka.htm

5. Schroeder, P. (2017) External Gravity Theory and Gravity "Bumps" during Eclipses. Journal of Physical Mathematics, 8, 2. 